\title{
Revue sur l'Ail et ses Composés Bioactifs
}

\author{
Banfitebiyi Gambogou, MSc \\ Yaovi A. Ameyapoh, PhD, Professeur
}

Laboratoire de Microbiologie et de Contrôle Qualité des Denrées Alimentaires (LAMICODA), Centre de Recherche et de Formation sur les Plantes Médicinales (CERFOPLAM), Université de Lomé, Lomé, Togo

\section{Holaly E. Gbekley, PhD}

Laboratoire de Microbiologie et de Contrôle Qualité des Denrées Alimentaires (LAMICODA), Centre de Recherche et de Formation sur les Plantes Médicinales (CERFOPLAM), Université de Lomé, Lomé, Togo

Laboratoire des Sciences Biomédicales, Alimentaires et Santé Environnementale (LaSBASE), Université de Lomé, Togo

\section{Bouraïma Djeri, PhD, Maitre-Assistant \\ Kouassi Soncy, PhD \\ Kokou Anani, PhD \\ Simplice D. Karou, PhD, Professeur}

Laboratoire de Microbiologie et de Contrôle Qualité des Denrées Alimentaires (LAMICODA), Centre de Recherche et de Formation sur les Plantes Médicinales (CERFOPLAM), Université de Lomé, Lomé, Togo

\section{Doi:10.19044/esj.2019.v15n6p74 ～URL:http://dx.doi.org/10.19044/esj.2019.v15n6p74}

\section{Résumé}

Introduction: Dans les pays en voie de développement, les problèmes du médicament se posent en termes d'insuffisance quantitative, qualitative et d'inaccessibilité économique. Pour pallier à ce problème, près de $80 \%$ de la population ont recours à la médecine traditionnelle. Cependant, la question n'est plus de démontrer l'efficacité de la médecine traditionnelle mais de s'en convaincre et convaincre les acteurs de la santé publique et des groupes de promotion de la santé. D'où notre objectif de vulgariser l'utilisation de l'ail par la population en montrant les composés bioactifs et les differentes formes d'ail et leur usage thérapeutique. Méthodologie: la recherche Littérature a été effectuée dans les bases de donnée de Medline, Google scholar, Research Gate, HINARI, Cochrane et livres en utilisant des mots clés tels que : Problématique des médicaments en Afrique, l'étude ethnobotanique, ethnomédecine, ethnopharmacologie, les composés bioactifs de l'ail. Cette recherche documentaire s'est faite sur la période du 08 juin 2018 au 23 
Novembre 2018. La recherche a été étendue sur les formes d'ail, les données cliniques de l'utilisation de l'ail. Synthèse: L'ail est utilisé depuis des millénaires tant pour ses talents culinaires que ses propriétés thérapeutiques. Les principaux composés responsables des vertus thérapeutiques sont les composés organosulfureux dont leur présence et concentration dépend de la forme d'ail utilisé. Conclusion: L'ail, permet de lutter contre certains germes infectieux de la peau et contre les parasites. Il est aussi utilisé dans le traitement de l'hypertension artérielle, de la coagulation du sang et l'hypercholestérolémie. Ainsi, il est recommandé de consommer des préparations conditionnées et standardisées de l'ail pour bénéficier de ses vertus.

Mots-clés: Ail, ethnomédecine, ethnopharmacologie, molécules bioactives, les différentes formes d'ail 


\title{
Review on Garlic and its Bioactive Compounds
}

\author{
Banfitebiyi Gambogou, MSc \\ Yaovi A. Ameyapoh, PhD, Professeur
}

Laboratoire de Microbiologie et de Contrôle Qualité des Denrées

Alimentaires (LAMICODA), Centre de Recherche et de Formation sur les Plantes Médicinales (CERFOPLAM), Université de Lomé, Lomé, Togo

\section{Holaly E. Gbekley, PhD}

Laboratoire de Microbiologie et de Contrôle Qualité des Denrées Alimentaires (LAMICODA), Centre de Recherche et de Formation sur les Plantes Médicinales (CERFOPLAM), Université de Lomé, Lomé, Togo

Laboratoire des Sciences Biomédicales, Alimentaires et Santé Environnementale (LaSBASE), Université de Lomé, Togo

\section{Bouraïma Djeri, PhD, Maitre-Assistant \\ Kouassi Soncy, PhD \\ Kokou Anani, PhD \\ Simplice D. Karou, PhD, Professeur}

Laboratoire de Microbiologie et de Contrôle Qualité des Denrées Alimentaires (LAMICODA), Centre de Recherche et de Formation sur les Plantes Médicinales (CERFOPLAM), Université de Lomé, Lomé, Togo

\begin{abstract}
Introduction: The availability and accessibility of medicines remains a real concern in developing countries. In the context of low economic income, most of the population (80\%) uses traditional medicine. The effectiveness of traditional medicine has been well established. This study suggests that public health actors and health promotion groups in developing countries use herbal medicine as an alternative to the problem of the effectiveness of chemical synthesis drugs. This paper focuses on evaluating the use of different forms of garlic by the population and the bioactive compounds of this plant. Methodology: A literature review was conducted in the Medline, Google Scholar, Research Gate, HINARI, and Cochrane databases using keywords such as Drugs in Africa, Ethnobotany, Ethnomedicine, Ethnopharmacology, Garlic Forms, and Compounds bioactives of garlic. Results: The use of garlic is as old as humanity especially in cooking and disease therapy. The main compounds responsible for the therapeutic virtues are the organosulfur compounds, and its concentration of which depends on the shape of the garlic.
\end{abstract}


Conclusion: Garlic can fight against skin infections, blood pressure drop, prevent blood clotting and hypercholesterolemia. Based on this study, it is recommended to consume conditioned and standardized garlic preparations in order to benefit from its virtues.

Keywords: Garlic, ethomedecine, ethnopharmacology, bioactives compounds, different forms of garlic

\section{Introduction}

Dans les pays en voie de développement, les problèmes du médicament se posent en termes d'insuffisance quantitative, qualitative et d'inaccessibilité économique (Baranyanka, 2015). Ces problèmes sont plus cruciaux en Afrique avec plus de 420 tonnes de produits médicaux de contrebande en Afrique de l'Ouest (Pharmacopoeia, 1992).

En Afrique, la médecine traditionnelle est en plein développement avec près de $80 \%$ des malades qui ont recours aux professionnels de la médecine traditionnelle pour les soins de santé primaires (Pharmacopoeia, 1992). Face à un public de plus en plus exigeant, la médecine traditionnelle doit convaincre si on veut l'utiliser comme alternatives thérapeutique. Cependant, la question n'est plus de démontrer l'efficacité de la médecine traditionnelle mais de s'en convaincre et convaincre les acteurs de la santé publique et des groupes de promotion de la santé. Ainsi, selon les recommandations de l'OMS et l'OUA qui préconise l'emploi de la pharmacopée traditionnelle et le concours de tous les professionnels de la médecine traditionnelle pour développer les soins de santé primaire afin de pallier le problème de pénurie des médicaments . Des études scientifiques sont menées par des institutions tels que l'I.R.S.S (Institut de Recherche en Science de la Santé) et PHYTOFLA du Burkina Faso, le DMT (Département de la Médecine Traditionnelle) du Mali, au Bénin la maison de la Feuille, et au Togo, CERFOPLAM (Centre de recherche et de Formation sur les Plantes Médicinales) et le Centre Omnithérapeutique Africain (COA). Les exploits de ces centres de recherche sur la médecine traditionnelle et les prouesses réalisées expliquent le regain d'intérêt des plantes médicinales (Gbekley et al., 2018).

Au Togo, le médicament reste un problème préoccupant (Yerima et al., 2015). La pénurie est constante dans les formations sanitaires due à la modestie des budgets. La faiblesse des ressources économiques des populations les limites dans l'achat des produits pharmaceutiques (Gnassingbe et al., 2018). Les conséquences sont l'automédication, le non-respect des prescriptions, utilisation anarchique des médicaments, ce qui entraine des résistances aux antibiotiques (Banfitebiyi et al., 2018a). Face à cette situation, 
les populations se retournent vers la médecine traditionnelle et ses produits dérivés (OMS, 2017).

Depuis bien longtemps, les Hommes traitent leurs maux avec les plantes. Parmi celles-ci, l'Allium sativum L. (ail), bien connu dans la population, comme possédant de nombreux bienfaits en passant par le traitement de l'insuffisance veineuse, l'hypertension, l'action antibactérienne, et bien d'autres (Gambogou et al., 2018; Gbekley et al., 2018). L'ail se retrouve sous différentes formes ayant divers composés plus ou moins concentrés. Cependant, la question n'est plus de démontrer l'efficacité de l'ail mais de montré les molécules bioactifs présents dans les différents types de préparation l'ail sous chaque formes afin de valoriser son utilisation en médicine traditionnelle.

\section{Méthodologie}

Nous avons effectué une revue documentaire sur la problématique des médicaments en Afrique, sur l'ail et les vertus thérapeutiques notamment ; les usages traditionnels par les populations et les activités biologiques et pharmacologiques au laboratoire. Une recherche systématique a été réalisée dans les bases de donnée Prota 4U, Pub Med, Hinari et Google Schoolar, Research Gate en utilisant les mots clés suivants : problématique des médicaments en Afrique de l'Ouest, ail, vertus thérapeutique de l'ail, ethnobotanique, ethnopharmacologie, composés bioactifs, activité biologique, donnée clinique. Cette recherche documentaire s'est faite sur la période du 08 juin 2018 au 23 Novembre 2018.

\section{Synthèse bibliographique}

Tableau 1. Identification de l'ail.

\begin{tabular}{|l|l|}
\hline Nom botanique & Allium sativum L. \\
\hline Famille & Lilliaceae \\
\hline Synonyme & \multicolumn{1}{|c|}{ Porvium sativum Relib } \\
\hline Noms communs & \multicolumn{1}{|c|}{ Anglais : Garlic } \\
\cline { 2 - 2 } & \multicolumn{1}{|c|}{ Français : Ail commun } \\
\hline Noms vernaculaires & $\begin{array}{l}\text { Burkina Faso : Mooré - Gando, Layi, Dioula - Laii, } \\
\text { Fulfuldé - Toumé }\end{array}$ \\
\cline { 2 - 2 } & $\begin{array}{l}\text { Ghana : Twi - Gyene Kankan, Ga Adangbe - Aya, Hausa - } \\
\text { Tafarmuwa }\end{array}$ \\
\cline { 2 - 2 } & Mali : Bambara - Tumé, Tamachek - Teskart \\
\cline { 2 - 2 } & $\begin{array}{l}\text { Nigéria : Hausa - Tafárnúúwáá, Igbo - Oy Ayón, Ayún, } \\
\text { Yoruba - Àlubósa, Ayúu }\end{array}$ \\
\cline { 2 - 2 } & Senegal: Wolof - Laji, Manding Bambara - Layi \\
\cline { 2 - 2 } & Togo: Ewe - Ayo, Kabyè - Ayo, Moba- Gabdjak \\
\hline
\end{tabular}




\section{Description de la plante}

Une herbe dressée, robuste et pérenne à bulbe de $60 \mathrm{~cm}$ de hauteur, avec un bulbe central, couvert d'écailles à l'aisselle. Le bulbe se compose d'un certain nombre de caïeux ou gousses enfermées dans une peau semblable à $\mathrm{du}$ papier. Les feuilles sont longues, plates et lisses avec une lame de feuille à forme cylindrique, creuse, linéaire, plate et solide à sommet aigu (apex acuminé); une inflorescence sphérique avec des fleurs colorées blanches ou rose-violacées sur des pédoncules grêles (Morrison, 1994).

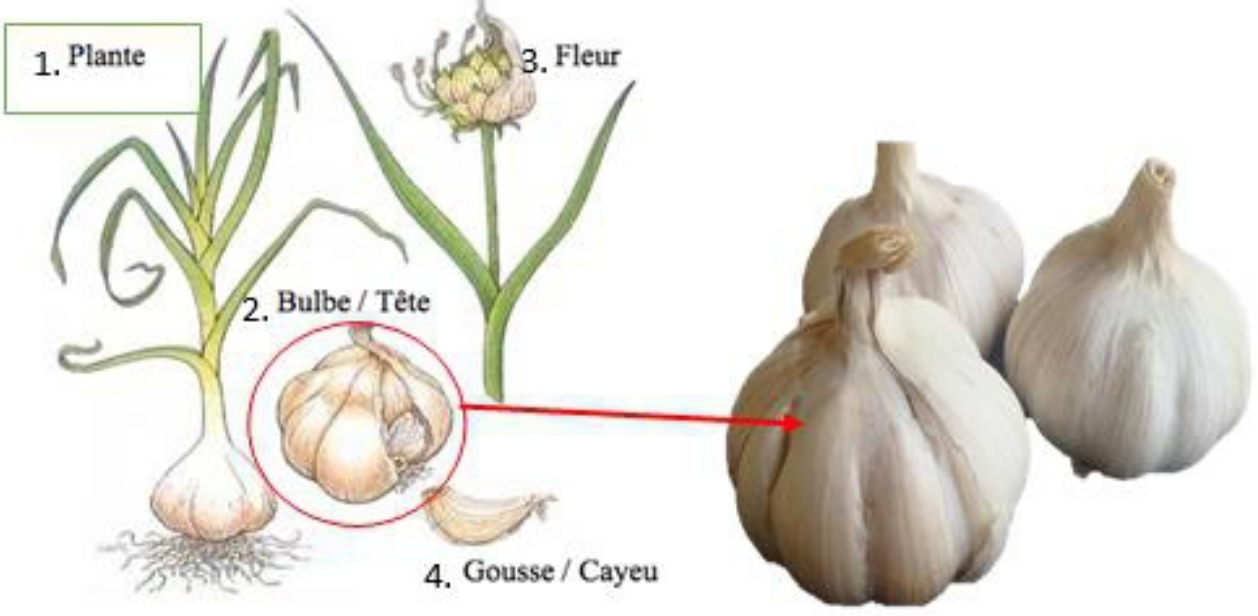

Figure 1. Présentation de l'Ail (Photo BioPhytoPharma)

\section{Habitat et répartition géographique}

A. sativum est originaire de l'Asie Centrale, mais cultivé maintenant dans de nombreuses parties du monde, notamment en Europe, en Afrique du Nord, en Asie, en Amérique du Nord et en Afrique de l'Ouest (Edouard, 1991; Morrison, 1994).

\section{Composition Biochimique de l'ail}

L'ail est une plante saine et complète qui jouit de propriétés médicinales très intéressantes. Il est utilisé autant en prévention de maladies que pour en guérir, il est composé en moyenne de $65 \%$ d'eau (contre plus de $85 \%$ pour la plupart des légumes frais), 27,5\% de glucides, $2 \%$ de protéines (Banfitebiyi et al., 2018a) et de 4,7\% de fibres et en contient des principes actifs tels que les composants soufrés. D'autre part, il regorge des vitamines A, B ou C, d'oligo-éléments tels que le sélénium, le cuivre, le fer et le magnésium et des acides aminés.

- Les glucides : sont composés des monosaccharides (fructose, glucose), des disaccharides (saccharose, lactose), des trisaccharides (raffinose), des tétrasaccharides (tétrafructose, scorodose), des 
polysaccharides (l'amidon, dextrine, inuline, fructosane), et autres comme le D-galactane, larabinose, pectines, D-fructane.

- Les lipides : la concentration en lipides dans l'ail est trop faible pour agir dans le corps. Il s’agit des acides gras (acide linoléique, acide linolénique, acide oléique, acide palmitique), des triglycérides, des phospholipides (phosphatidylcholine, phosphatidylsérine, phosphatidyléthanolamine), des prostaglandines (prostaglandine A, prostaglandine $\mathrm{E}$, prostaglandine $\mathrm{F}$ ).

- Les composés soufrés : 2,3\% de composés soufrés. Alliine, allicine $\left(\mathrm{C}_{6} \mathrm{H}_{10} \mathrm{OS}_{2}\right)$ et les dérivés d'allicine (trisulfures divers, les ajoènes, disulfure de diallyl). Ils sont à l'origine de la plupart des vertus pharmacologiques. Les principaux composés oragnosulfrés présents dans le bulbe de l'ail sont : l'alliine (S-allylcystéine sulfoxide), la Vglutamyl-S-allylcystéine, la méthiin (S-méthylcystéine sulfoxyde), l'isoalliin (S-trans-1-propenylcystéine sulfoxyde) (Anton, 2016).

- Les protides : les protides regroupent les protéines et les acides aminés (la lysine, la thréonine, la valine, la méthionine, l'isoleucine, le tryptophane, la phénylalanine, la leucine, l'histidine, l'arginine, l'acide aspartique, la sérine, la glutamine, la proline, la glycine, l'alanine et la cystéine).

- Fibres alimentaires : les fibres alimentaires sont les parties d'origine végétale non transformées par les enzymes de la digestion. Ce sont des substances résiduelles provenant de la paroi cellulaire ou le cytoplasme des végétaux, constituées de mélanges complexes de glucides.

- Les minéraux et oligo-éléments : le phosphate, le potassium, le magnésium, le cuivre, le fer, le manganèse, le zinc et le sélénium. L'ail possède une grande quantité de sélénium contrairement aux autres légumes : diméthylsélénide, acide méthyle-ster-méthane-sulfénosélénoïque, diméthyldisélénide, bi-(méthylthio)-sélénide, allylméthylsélénide, acide méthylester-2-propènesulfénosélénoïque, acide propylester-1-propènesulfénosélénoïque, allylthiométhylthiosélénide (Anton, 2016).

- Les vitamines : les vitamines A, B1, B2, B6, C et E.

- Quelques traces de pigments : comme de la chlorophylle, des caroténoïdes, des anthocyanes (ce sont des pigments hydrosolubles qui donnent une coloration rouge violette ou bleue). Les quantités des pigments sont faibles dans l'ail, il n'est pas coloré.

- Autres composés divers : des acides comme l'acide phénol, l'acide organique, les saponosides, les flavonoïdes, les phytohémagglutinines, les gibbérellines A3 et A7. 


\section{Les différents types d'ail}

Des lors, se pose le problème du mode de préparation et de la composition des extraits qui sont utilisés dans les tests pharmacologiques. En effet, il n'existe pas d'essence d'ail qui soit stockée et secrétée par des organes caractéristiques et la présence de telle ou telle molécule dans les extraits dépend de leur mode de préparation. Les produits commerciaux de l'ail se présentent sous diverses formes:

- L'huile essentielle d'ail : obtenue par distillation. Une gousse d'ail contient 0,2 à $0,5 \%$ d'huile essentielle, avec de nombreux composés soufrés comme le disulfure de diallyl ou le trisulfure de diallyl. Les gousses d'ail sont d'abord broyées dans l'eau puis distillées ou extraites grâce à un solvant organique, comme l'hexane qui permet d'obtenir la fraction d'huile. Les composés solubles dans l'eau, y compris l'allicine, sont éliminés. Des capsules d'ail sont disponibles contenant de l'huile végétale avec une petite quantité d'huile essentielle, puisque l'odeur est très prenante (Feng et al., 2018).

- La poudre d'ail : La poudre d'ail déshydratée est généralement utilisée comme condiment dans les aliments. Les gousses d'ail doivent être pulvérisées, broyées, séchées pour en extraire la poudre. Le principal composé contenu dans la poudre d'ail et dans l'ail frais est l'alliine. Elle contient certains constituants similaires à ceux de l'ail cru, même si les concentrations peuvent varier considérablement. De plus, l'inactivation de l'alliinase se fait lorsque le $\mathrm{pH}$ est inférieur à 3 . Il est totalement décomposé à $20^{\circ} \mathrm{C}$ en 20 heures (Mercier-Fichaux, 2016).

- Le macérât ou l'extrait d'huile d'ail est également utilisé comme condiment. Le macérât d'huile est fait de mélanges de gousses d'ail entièrement broyées encapsulées dans l'huile végétale. Pendant le processus de fabrication, l'alliine peut être convertie en allicine. Comme l'allicine est instable, il se décompose rapidement, le macérât contient principalement des composés tels que les vinyldithiines, les ajoènes (Joshi et al., 1987).

- L'extrait d'ail vieilli : Une solution d'extraction composée d'eau distillée et diluée dans 15-20\% d'éthanol doit être utilisée pour extraire l'ail dit vieilli. Cet extrait doit être âgé d'au moins 10 mois à température ambiante. Ce stockage conduit à une altération de la composition de l'ail. Pendant ce processus, l'odeur, les composés irritants sont convertis en composés soufrés stables et sûrs. Les composés d'ail sont des composés solubles dans l'eau et une petite quantité de composé soluble dans l'huile. Les composés solubles dans l'eau sont S-Allyle-Cystéine (SAC) et S-Allyle- Mercaptocystéine, 
avec une perte importante de l'activité de l'allicine. L'extrait d'ail vieilli est très utilisé.

Il est donc indispensable de connaitre la composition d'un produit d'ail avant d'entreprendre toute étude pharmacologique.

\section{Utilisations ethnomédicales}

L'ail est un anti-cholestérol, un anti-hypertenseur, un anticoagulant, un anti-diarrhéique, un anti-dysentérique, un stimulant immunitaire, un stomachique, un sudorifique, un expectorant, un vermifuge, un anti-irritant, un diurétique, un antibiotique à large spectre. Il est utilisé en externe pour l'arthrite, les cors, les verrues, les névralgies (Edouard, 1991; Lecerf, 2016), la fièvre, la toux, les flatulences, l'ulcère, la raucité de la voix, la bronchite et autres problèmes respiratoires, les maladies de la peau, les brûlures, l'otite et l'amygdalite, les rhumatismes, la tuberculose, la typhoïde, l'artériosclérose, le diabète, l'hyperlipidémie et la prévention de l'athérosclérose (en fonction de l'âge) des changements vasculaires (Mercier-Fichaux, 2016; WHO, 1999).

\section{Activités biologiques et pharmacologiques}

Plusieurs études scientifiques ont montré que l'ail a des propriétés antihyperlipidémiantes, antihypertensives et anticoagulantes (Auer et al., 1990; Barrie, 1987; Bordia et al., 1998; Gebhardt et al., 1994; Goetz \& Wuyts, 2008; Sayouri et al., 2015). De nombreuses actions thérapeutiques de l'ail sont attribuées à l'allicine et à ses métabolites. Par exemple l'allicine et son dérivé sulfurique ont la propriété d'inhibé in vitro la prolifération de la leucémie maligne de plusieurs cellules humaines non atteintes (Abdullah, 1989). Des études in vitro ont montré que l'ajoène possède des propriétés antithrombotiques et antimicrobiennes en réduisant le taux de cholestérol; l'ajoène présente des effets inhibiteurs sur l'activation des plaquettes, leurs liaisons à la paroi du vaisseau sanguin endommagé et à la formation de thrombus (Apitz-Castro et al., 1992; Apitz-Castro et al., 1994; Apitz-Castro et al., 1986). Il prévient également la perte de plaquettes sanguines, empêche la circulation de la lipoxygénase et l'activité de la tyrosine phosphatase dans les plaquettes humaines (Srivastava \& Tyagi, 1993) de même que la réduction de la biosynthèse du cholestérol (Gebhardt et al., 1994). Les composés de disulfure et de trisulfure de diallyle possèdent des propriétés d'agrégation plaquettaire et de formation antithromboxane (Bordia et al., 1998). Des extraits aqueux et organiques de l'ail ont inhibé in vivo l'agrégation plaquettaire. Les extraits d'ail réduisent l'accumulation de cholestérol dans les vaisseaux sanguins et le développement de plaques artériosclérotiques dans la paroi artérielle de lapins nourris en cholestérol (Efendy et al., 1997; Koscielny et al., 1999). Les extraits ont également montré des effets antihypertenseurs, 
une activité anticoagulante accrue, une diminution de la viscosité du sang et l'amélioration de la fonction cardio-vasculaire (Ernst, 1987; Kendler, 1987). L'huile d'ail produit inversion marquée de changements métaboliques liés à l'infarctus du myocarde induit par l'isoprotérénol (Saravanan \& Prakash, 2004; Sayouri et al., 2015). Les extraits d'ail ont montré des propriétés larvicides contre les larves d'anophèles et une forte activité inhibitrice contre une série de bactéries pathogènes et champignons (Benkeblia, 2004). Ajoène présente des activités antimycosiques, antimicrobiennes et antivirales (Davis \& Perrie, 2003; El-Dougdoug et al., 2018). D'autres tests in vitro et in vivo ont également montré que l'ail a des effets antifongiques à large spectre (Benkeblia, 2004; Davis \& Perrie, 2003; Tansey \& Appleton, 1975) et présente une activité synergique avec l'amphotéricine B qui inhibe la croissance fongique (Tansey \& Appleton, 1975). De vastes recherches scientifiques ont montré que divers produits commerciaux faits à base d'ail possèdent des activités antivirales contre une série de virus, y compris l'herpès simplex de types 1 et 2, la grippe A et les virus de type B, le cytomégalovirus humain, le virus de la stomatite vésiculeuse, le rhinovirus, le virus de l'immunodéficience humaine (VIH), la pneumonie virale et le rotavirus (Faik, 2018; Goetz \& Wuyts, 2008). Il a été démontré que l'allicine a une activité antibactérienne (Cavallito \& Bailey, 1944; Gambogou et al., 2018). De nombreuses études épidémiologiques, cliniques et de laboratoire ont démontré le rôle de l'ail dans la prévention du cancer. Les constituants chimiques de la poudre et de l'huile d'ail possèdent un puissant effet antibactérien sur Helicobacter pylori, qui peut expliquer son effet supposé protecteur contre le cancer gastrique (Bianchini \& Vainio, 2001; Le Bon, 2016). Les propriétés chimio-préventives de la plante ont été attribuées à des composés organosulfuriques qui modulent l'activité de plusieurs enzymes du métabolisme, en activant ou détoxifiant les agents cancérigènes et en inhibant la formation de plusieurs additifs d'ADN dans les tissus cibles. Il a été démontré que le disulfure de diallyle possédait une puissante activité chimio-préventif contre les cancers du côlon, du poumon et de la peau (Le Bon, 2016).

\section{Données cliniques}

Les études mettent cependant en évidence que les effets protecteurs de l'ail (protection cardiovasculaire, prévention des cancers du côlon et de la prostate par exemple) ne sont observés que dans le cadre d'une alimentation globalement équilibrée associée à la consommation quotidienne de deux gousses d'ail cru par jour, soit 6 g/j (Lecerf, 2016). Or les données disponibles montrent que dans les pays occidentaux les quantités d'ail consommé sont beaucoup plus faibles : seulement $15 \%$ de la population britanniques consomment 6 g d'ail/semaine (Mercier-Fichaux, 2016) et à peine $20 \%$ des Américaines ingèrent plus de $2 \mathrm{~g}$ d'ail/semaine (Mercier-Fichaux, 2016). Au 
Togo, $43.6 \%$ de la population consomment de $5 \mathrm{~g}$ d'ail/jour (Banfitebiyi et al., 2018b).

Les préparations de la poudre d'ail ont montré un potentiel de réduction des lipides et une diminution du taux d'activateur tissulaire du plasminogène et d'hématocrite. Ce qui a entrainé une augmentation de 4,2\% le diamètre moyen des artérioles et de 5,9\% les veinules par rapport aux contrôles, une augmentation du débit érythrocytaire capillaire et la diminution du taux de viscosité et de fibrinogène du plasma. L'agrégation plaquettaire induite par l'adénosine diphosphate et le collagène a été significativement inhibée entre 2 à 4 heures après l'ingestion d'ail et, est restée inférieure pendant 7 à 14 jours après le traitement. La consommation d'ail à induit une diminution de la proportion des agrégats de circulation plaquettaire et l'agrégation spontanée de plaquettes par rapport au groupe placebo et a diminué également la glycémie moyenne (WHO, 1999). Plusieurs rapports cliniques et des métaanalyses ont révélé les effets anti-cholestérol de l'ail cru et de suppléments d'ail. Il a été montré que l'ail peut diminuer les lipoprotéines de faible densité et augmenter les niveaux de lipoprotéines de haute densité (Chang \& Johnson, 1980; Ernst, 1987). L'application topique de l'ajoène a produit une réponse clinique significative chez les patients atteints d'un carcinome basocellulaire de la peau. L'ail présente un pouvoir stimulant de cellules effectrices immunisées, comprenant les cellules T et celles de morts naturelles (Bianchini \& Vainio, 2001).

\section{Actions thérapeutiques}

Antihypertenseur, antidiabétique, anti-thrombotique, antifongique, antioxydant, anticancérigéne, antibactérienne, anti-asthmatique, immunomodulateur, digestif, anti-inflammatoire, antipyrétique, antiscorbutique, antitussif, expectorant, myorelaxant GIT lisse, antibactérien, anticoagulant, carminative, diaphorétique, antihyperhomocysteinemique, antihyperlipidémiant, stomachique (Corzo-Martínez et al., 2007; Gambogou et al., 2018; Goetz \& Wuyts, 2008).

\section{Les composés organosulfurés responsables des vertus thérapeutiques de l'ail}

L'ail est utilisé depuis des millénaires tant pour ses talents culinaires que ses propriétés thérapeutiques. C'est une plante aux multiples vertus (Lecerf, 2016). L'ail et d'autres plantes de la famille Allium sont connus pour leurs vertus thérapeutiques depuis des siècles et de nombreuses propriétés pharmacologiques (hypocholestérolémiante, anticoagulante, anti-oxydante, antibactérienne, antivirale et anticancérogène) sont attribuées à cette famille de plantes (Faiza, 2016). 
Le principal composé soufré, l'alliine, est une substance végétale pratiquement dépourvue d'odeur, présente dans le bulbe d'ail frais intact (Lenková et al., 2018). L'alliine est obtenu au cours de l'hydrolyse et l'oxydation de la $\mathrm{V}$-glutamyl-S-allylcystéine, cette réaction est catalysée par la V-glutamyl transpeptidase (Oosthuizen et al., 2018). L'alliine (Sallylcystéine) (composé stable et inodore) inhibe la prolifération des mélanomes dans des lignées cellulaires humaines ou elle induit également une diminution de gangliosides à la surface des cellules tumorales (Le Bon, 2016). Les $\mathrm{V}$-glutamylpeptides et Ia $\mathrm{V}$-glutamylallylcysteine sulfoxyde pourraient exercer un effet bénéfique sur l'hypertension (Goetz \& Wuyts, 2008).

Lorsque la gousse d'ail crue est fraîchement coupée ou broyée, l'alliine entre en contact avec l'enzyme vacuolaire alliinase qui lyse les cystéines sulfoxides cytosoliques, et se convertit en allicine qui confère son odeur si particulière à l'ail, et surtout ses propriétés pharmacologiques et thérapeutiques, notamment antibactériennes (Faik, 2018). Rapidement oxydée à l'air, l'allicine se transforme elle-même en présence d'eau en polysulfure d'allyle tels que le sulfure de diallyle (SDA), le disulfure de diallyle (DSDA), le trisulfure de diallyle (TSDA), et le tétrasulfure de diallyle (TTSDA). Ces molécules sont responsables de l'odeur fortement marquée de l'essence d'ail. L'allicine est le principe actif responsable de l'activité antiseptique, antibactérienne, antivirale, antifongique, antiparasitaire, bactéricide et bactériostatique de l'ail (Oosthuizen et al., 2018). Il a été montré que le jus d'ail dilué 125000 fois avait des propriétés inhibitrices sur le développement des entérobactéries, Staphylocoques, des Streptocoques (Gambogou et al., 2018), des Vibrio, des Bacillus et une activité sur certains champignons zoopathogènes et de nombreuses levures (Tansey \& Appleton, 1975). Il a prouvé son activité antifongique sur Microsporum, Tricophyton, Candida, Cryptococcus et Aspergillus (Tansey \& Appleton, 1975). Les études de l'Institut des Plantes Médicinales de Poznan (Pologne) ont montré son action importante sur les dermatophytes (Lecerf, 2016). L'ail est utilisé depuis pour ses propriétés antivirales dans le traitement de la méningite encéphalite virale aiguë et de la méningite à Cryptococcus, et des vers intestinaux (Benkeblia, 2004; Davis \& Perrie, 2003).

L'association de sulfures organiques avec un produit non sulfuré présente les caractères d'un alcaloïde. Ce principe actif (alcaloïde) dépourvu de soufre, est inactif par lui-même et n'acquiert son pouvoir hypoglycémiant que par l'association avec les composés sulfurés du suc d'ail ou la combinaison aux sulfures d'allyle ou de diallyle purs (Cavallito \& Bailey, 1944). Le disulfure d'allyle, le trisulfure d'allyle et surtout le trisulfure de méthylallyle ont cette même propriété en inhibant la thromboxane-synthétase. L'allicine, quant à elle, a un rôle sur les plaquettes, les neutrophiles par un mécanisme faisant intervenir les ions calcium (Lenková et al., 2018). Les fructosanes et 
disulfure d'allyle agiraient comme vasodilatateur des artérioles et des capillaires ce qui produirait une hypotension (Saravanan \& Prakash, 2004).

La formation de l'ajoène est favorisée quand l'allicine est dissoute dans certains solvants (Apitz-Castro et al., 1986). Il est plus abondant et stable dans un macérât d'ail haché dans de l'huile d'olive que dans l'ail simplement écrasé. La cuisson transforme rapidement, en quelques minutes, tous ces composés soufrés en produits de condensation cyclique (vinyldithiine). Il est donc impératif d'utiliser l'ail cru fraîchement écraser ou macéré dans de l'huile pour bénéficier au maximum de ses vertus médicinales telles que la protection cardiovasculaire, la prévention des cancers du côlon et de la prostate par exemple. Les ajoènes auraient une activité contre Candida albicans, et un effet antiagrégant plaquettaire, de plus, ils inhibent la synthèse de thromboxane (Bérénice, 2012).

Il a été également montré que l'huile essentielle d'ail dont le sulfure de diallyle a été converti en bromure d'allyle a des effets carminatifs. L'ail est aussi cholérétique et cholagogue (Boumaza \& Belal, 2017).

\section{Conclusion}

L'ail favorise la baisse de la pression artérielle, de la coagulation et du taux de cholestérol, d'où ses effets bénéfiques au niveau cardio-vasculaire. Ses propriétés, permettant de lutter contre certains germes infectieux de la peau et contre les parasites. Ainsi, pour obtenir un maximum de bénéfices avec un minimum de risques, il est conseillé de consommer des préparations conditionnées et standardisées, aux doses quotidiennes préconisées. En cas de préparations faites maison, il est également nécessaire de respecter les indications.

\section{Conflits d'intérêts}

Les auteurs déclarent qu'il n'y a aucun conflit d'intérêts.

\section{Contributions des auteurs}

Tous les auteurs ont contribué à la conduite de ce travail. Les auteurs de cet article ont lu et approuvé son contenu.

\section{References:}

1. Abdullah, T. (1989). Enhancement of natural killer cell activity in AIDS with garlic. Journal of Oncology 21, 52-53.

2. Anton, R. (2016). Les constituants spécifiques des AlliaceaeThe specific constituents of Alliaceae. Phytothérapie 14, 149-153.

3. Apitz-Castro, R., Badimon, J.J., \& Badimon, L. (1992). Effect of ajoene, the major antiplatelet compound from garlic, on platelet thrombus formation. Thrombosis research 68, 145-155. 
4. Apitz-Castro, R., Badimon, J.J., \& Badimon, L. (1994). A garlic derivative, ajoene, inhibits platelet deposition on severely damaged vessel wall in an in vivo porcine experimental model. Thrombosis research 75, 243-249.

5. Apitz-Castro, R., Escalante, J., Vargas, R., \& Jain, M.K. (1986). Ajoene, the antiplatelet principle of garlic, synergistically potentiates the antiaggregatory action of prostacyclin, forskolin, indomethacin and dypiridamole on human platelets. Thrombosis research 42, 303-311.

6. Auer, W., Eiber, A., Hertkorn, E., Hoehfeld, E., Koehrle, U., Lorenz, A., Mader, F., Merx, W., Otto, G., \& Schmid-Otto, B. (1990). Hypertension and hyperlipidaemia: garlic helps in mild cases. British journal of clinical practice. Supplement 69, 3-6.

7. Banfitebiyi, G., Kokou, A., Simplice, D.K., Yaovi, A.A., \& Simpore, J. (2018a). Effect of Aqueous garlic extract on biofilm formation and antibiotic susceptibility of multidrug-resistant uropathogenic Escherichia coli clinical isolates in Togo. International Journal Advanced Multidisciplinary Research 5, 23-33.

8. Banfitebiyi, G., Raouf, T.M., Djeri , B., Messanh D.K., Simplice, D.K, \& Yaovi A.A. (2018b). Assessment of Knowledge and Consumption Patterns of Garlic and the use of phytotherapy/aromatherapy in treatment of diseases in Togo. World Journal of Pharmaceutical Research 7, 12.

9. Baranyanka, P. (2015). La problématique des brevets et de l'accès aux médicaments dans les pays en développement par l'approche des biens publics mondiaux.

10. Barrie, S.A. (1987). Effects of garlic oil on platelet aggregation, serum lipids, and blood pressure in humans. J. Orthomol. Med. 2, 15-21.

11. Benkeblia, N. (2004). Antimicrobial activity of essential oil extracts of various onions (Allium cepa) and garlic (Allium sativum). LWT-food science and technology 37, 263-268.

12. Bérénice, D. (2012). Production de dérivés de l'alliine de l'ail (Allium sativum L.) présentant des propriétés thérapeutiques.

13. Bianchini, F., \& Vainio, H. (2001). Allium vegetables and organosulfur compounds: do they help prevent cancer? Environmental health perspectives 109, 893.

14. Bordia, A., Verma, S., \& Srivastava, K. (1998). Effect of garlic (Allium sativum) on blood lipids, blood sugar, fibrinogen and fibrinolytic activity in patients with coronary artery disease. Prostaglandins, leukotrienes and essential fatty acids 58, 257-263.

15. Boumaza, I., \& Belal, S. (2017). Effet du traitement thermique et chimique sur la physico-chimie de la pâte d'Ail. 
16. Cavallito, C.J., \& Bailey, J.H. (1944). Allicin, the antibacterial principle of Allium sativum. I. Isolation, physical properties and antibacterial action. Journal of the American Chemical Society 66, 1950-1951.

17. Chang, M.L.W., \& Johnson, M.A. (1980). Effect of garlic on carbohydrate metabolism and lipid synthesis in rats. The Journal of nutrition 110, 931-936.

18. Corzo-Martínez, M., Corzo, N., \& Villamiel, M. (2007). Biological properties of onions and garlic. Trends in food science \& technology 18, 609-625.

19. Davis, S., \& Perrie, R. (2003). The in-vitro susceptibility of Cryptococcus neoformans to allitridium, Program and Abstracts of the 15 th Congress of ISHAM (abstract 113). San Antonio, TX, USA, May $25 \mathrm{e} 29$.

20. Edouard, A. (1991). Contribution to ethnobotanical and floristic studies in Western Nigeria. Organization of African Unity, Scientific Technical \& Research Commission.

21. Efendy, J.L., Simmons, D.L., Campbell, G.R., \& Campbell, J.H. (1997). The effect of the aged garlic extract,Kyolic', on the development of experimental atherosclerosis. Atherosclerosis 132, 37 42.

22. El-Dougdoug, K.A., Sofy, A.R., Mousa, A.A., Sofy, M.R., Hmed, A.A., \& Abbas, A.A. (2018). Safe and Efficacious AntiCytomegalovirus Agents with Therapeutic Activity in vitro. Journal of Microbiology Research 8, 33-42.

23. Ernst, E. (1987). Cardiovascular effects of garlic (Allium sativum): a review. Pharmatherapeutica 5, 83-89.

24. Faik, G. (2018). The inhibition effect of garlic-derived compounds on human immunodeficiency virus type 1 and saquinavir. Journal of biochemical and molecular toxicology, e22215.

25. Faiza, M. (2016). Valorisation des plantes condimentaires cultivées et spontanées dans l'ouest algérien cas du genre Allium.

26. Feng, B., Hui, R.-j., Tu, Y.-f., Wang, J.-f., \& Xuan, J.-g. (2018). Garlic essential oil provides lead discharging effect on human body: An efficacy and mechanism study. Bioactive Compounds in Health and Disease 1, 172-173.

27. Gambogou, B., Ouattara, A.K., Taale, E., Karou, S.D., Ameyapoh, Y.A., \& Simpore, J. (2018). Garlic as Alternative Therapy to Treat Uropathogene Bacteria in Women with Urinary Tract Infection in Lomé, Togo. Eurpean Journal of Pharmaceutical and Medical Research 5, 7 . 
28. Gbekley, H.E., Karou, S.D., Katawa, G., Tchacondo, T., Batawila, K., Ameyapoh, Y., \& Simpore, J. (2018). Ethnobotanical survey of medicinal plants used in the management of hypertension in the Maritime region of Togo. African Journal of Traditional, Complementary and Alternative Medicines 15, 85-97.

29. Gebhardt, R., Beck, H., \& Wagner, K.G. (1994). Inhibition of cholesterol biosynthesis by allicin and ajoene in rat hepatocytes and HepG2 cells. Biochimica et Biophysica Acta (BBA)-Lipids and Lipid Metabolism 1213, 57-62.

30. Gnassingbe, A., Flahault, A., Geissbuhler, A., Sprumont, D., \& Awesso, A. (2018). État des lieux de la lutte contre le marché informel de médicaments au Togo: dispositifs et limites. Médecine et Santé Tropicales 28, 5-11.

31. Goetz, P., \& Wuyts, D. (2008). Phytothérapie et nutrithérapie de l'hypertension artérielle. Phytothérapie 6, 247-252.

32. Joshi, D., Dikshit, R., \& Mansuri, S. (1987). Gastrointestinal actions of garlic oil. Phytotherapy Research 1, 140-141.

33. Kendler, B.S. (1987). Garlic (Allium sativum) and onion (Allium cepa): a review of their relationship to cardiovascular disease. Preventive medicine 16, 670-685.

34. Koscielny, J., Klüssendorf, D., Latza, R., Schmitt, R., Radtke, H., Siegel, G., \& Kiesewetter, H. (1999). The antiatherosclerotic effect of Allium sativum. Atherosclerosis 144, 237-249.

35. Le Bon, A.-M. (2016). Alliacées et prévention des cancers. Phytothérapie 14, 159-164.

36. Lecerf, J.-M. (2016). Ail et santé. Phytothérapie 14, 139-139.

37. Lenková, M., Bystrická, J., Chlebo, P., \& Kovarovič, J. (2018). Garlic (Allium sativum L.)-the content of bioactive compounds. Potravinarstvo Slovak Journal of Food Sciences 12, 405-412.

38. Mercier-Fichaux, B. (2016). L'ail un alicament qui a du piquant! Garlic as a functional food and spice! Phytothérapie 14, 176-180.

39. Morrison, B.H. (1994). The useful plants of west tropical Africa. Volume 2: Families EI. Royal Botanic Gardens.

40. OMS. (2017). Évaluation et examen de la Stratégie mondiale et du Plan d'action pour la santé publique, l'innovation et la propriété intellectuelle. Organisation mondiale de la Santé.

41. Oosthuizen, C.B., Reid, A.-M., \& Lall, N. (2018). Garlic (Allium sativum) and Its Associated Molecules, as Medicine, Medicinal Plants for Holistic Health and Well-Being. Elsevier, pp. 277-295.

42. Pharmacopoeia, G.H. (1992). Policy Research and Strategic Planning Institute (PORSPI). The Advent Press. Accra. 
43. Saravanan, G., \& Prakash, J. (2004). Effect of garlic (Allium sativum) on lipid peroxidation in experimental myocardial infarction in rats. Journal of ethnopharmacology 94, 155-158.

44. Sayouri, H., Boudier, A., Vigneron, C., Leroy, P., \& Le Tacon, S. (2015). Le sulfure d'hydrogène: une thérapeutique d'avenir dans la neuroprotection post-arrêt cardiorespiratoire?, Annales Pharmaceutiques Françaises. Elsevier, pp. 401-410.

45. Srivastava, K., \& Tyagi, O. (1993). Effects of a garlic-derived principle (ajoene) on aggregation and arachidonic acid metabolism in human blood platelets. Prostaglandins, leukotrienes and essential fatty acids 49, 587-595.

46. Tansey, M.R., \& Appleton, J.A. (1975). Inhibition of fungal growth by garlic extract. Mycologia 67, 409-413.

47. WHO (1999). WHO monographs on selected medicinal plants. World Health Organization.

48. Yerima, M., Ekouevi, D., Tchounga, B., Afanvi, A., Potchoo, Y., Kassankogno, Y., \& Napo-Koura, F. (2015). Acces au traitement anticancereux au Togo (access to treatment of cancer in Togo). Journal de la Recherche Scientifique de l'Université de Lomé 17, 279-288. 